University of Wollongong

Research Online

Faculty of Engineering and Information

Faculty of Engineering and Information

Sciences - Papers: Part B

Sciences

2018

\title{
A holistic approach for integrated volt/var control in MV and LV networks
}

Nurul Izzah Binti Mohammad Afandi

University of Wollongong, nibma972@uowmail.edu.au

Phil Ciufo

Ashish P. Agalgaonkar

University of Wollongong, ashish@uow.edu.au

Sarath Perera

University of Wollongong, sarath@uow.edu.au

Follow this and additional works at: https://ro.uow.edu.au/eispapers1

Part of the Engineering Commons, and the Science and Technology Studies Commons

Research Online is the open access institutional repository for the University of Wollongong. For further information contact the UOW Library: research-pubs@uow.edu.au 


\title{
A holistic approach for integrated volt/var control in MV and LV networks
}

\author{
Abstract \\ Voltage regulation in medium voltage (MV) and low voltage (LV) distribution networks has always been \\ considered and approached as separate issues. However, changes in voltage magnitudes in an MV \\ network can affect voltage magnitudes in an associated LV network and vice versa. In addition, voltage \\ changes in an MV network can be contrary to changes in the associated LV network. This paper presents \\ a voltage regulation methodology for MV and LV networks as a combined, network-wide problem. A \\ theoretical formulation is developed to present MV and LV networks in a holistic manner, applicable for \\ the development of an efficient voltage regulation approach. The proposed formulation is tested on a \\ modified IEEE 13 node test feeder and verified using OpenDSS. The results confirm that the proposed \\ formulation can successfully solve both MV and LV networks simultaneously. Based on the developed \\ theoretical formulation, this paper proposes an integrated volt/var control philosophy to perform voltage \\ regulation in both MV and LV networks jointly. The proposed volt/var control method is tested on the \\ same test feeder over a 24 -h period with varying load and generation. The results confirm that the \\ proposed volt/var control method can successfully improve voltage profiles across both MV and LV \\ networks in comparison to using a conventional volt/var control method.

\section{Disciplines} \\ Engineering | Science and Technology Studies \\ Publication Details \\ I. Afandi, P. Ciufo, A. Agalgaonkar \& S. Perera, "A holistic approach for integrated volt/var control in MV \\ and LV networks," Electric Power Systems Research, vol. 165, pp. 9-17, 2018.
}




\title{
A Holistic Approach for Integrated Volt/Var Control in MV and LV Networks
}

\author{
Izzah Afandi, Philip Ciufo, Ashish Agalgaonkar, Sarath Perera \\ School of Electrical, Computer and Telecommunications Engineering, University of Wollongong, Wollongong, NSW, \\ Australia
}

\begin{abstract}
Voltage regulation in medium voltage (MV) and low voltage (LV) distribution networks has always been considered and approached as separate issues. However, changes in voltage magnitudes in an MV network can affect voltage magnitudes in an associated LV network and vice versa. In addition, voltage changes in an MV network can be contrary to changes in the associated LV network. This paper presents a voltage regulation methodology for MV and LV networks as a combined, network-wide problem. A theoretical formulation is developed to present MV and LV networks in a holistic manner, applicable for the development of an efficient voltage regulation approach. The proposed formulation is tested on a modified IEEE 13 node test feeder and verified using OpenDSS. The results confirm that the proposed formulation can successfully solve both MV and LV networks simultaneously. Based on the developed theoretical formulation, this paper proposes an integrated volt/var control philosophy to perform voltage regulation in both MV and LV networks jointly. The proposed volt/var control method is tested on the same test feeder over a 24-hour period with varying load and generation. The results confirm that the proposed volt/var control method can successfully improve voltage profiles across both MV and LV networks in comparison to using a conventional volt/var control method.
\end{abstract}

Keywords: Medium voltage network, low voltage network, voltage regulation, volt/var control

\section{Introduction}

The use of renewable energy sources in distribution networks all around the world has been progressing rapidly in recent years, contributing to active distribution networks. This progress, however, has added some complexities that can challenge traditional voltage regulation methods in distribution networks. For example, voltage regulators are utilised in low voltage (LV) networks to regulate the voltage level in the network, however this has been proven to be a challenge since many of such devices do not allow reverse power flow that may come as a consequence of increasing photovoltaic (PV) system penetration [1]. In addition, voltage regulation is traditionally performed in the medium voltage (MV) side of the network only, and most PV systems are installed in LV networks, challenging the traditional paradigm of unidirectional power flow in distribution networks.

Due to the complex nature of an LV network, voltage control methods implemented on the MV side of a network may not be adequate to improve the voltage profile on the LV network. One 
method employed by distribution network service providers (DNSPs) is volt/var control which integrates and adjusts voltage and reactive power control to perform voltage regulation. The authors of [2-4] proposed different volt/var control methods which generally accomplish the desired voltage regulation performance, however these methods are carried out in the MV network only, which may not necessarily improve the voltage on the LV network.

Voltage control methods in the LV network are either non-existent where it is supposed to be governed by the control strategy in the associated MV network or exists solely on its own where it is supposed to not have any influence on the associated MV side [5-7]. Nonetheless, changes applied to one side of the network can influence the other, and voltage control methods which are segregated between MV and LV networks may not address this properly. In other words, there is a fundamental disconnect between voltage regulation in distribution networks because of this segregated approach. This disconnect is prevalent in active distribution networks since issues such as voltage rise and reverse power flow are becoming commonplace [8]. This shows that a voltage regulation method that combines both MV and LV networks by taking into account challenges posed in both sides of the distribution network while efficiently utilising voltage regulating resources available throughout the entire network is important.

An efficient voltage regulation method delivers the design requirements for a distribution network which in turns relies on the understanding of the state of the network at any particular instance, including current flow and voltage drop. Load flow methods can be used to solve for unknown voltages in a distribution network. Once these voltages are known, other parameters such as current flow and active and reactive power flows can be calculated. There are a wide variety of load flow algorithms developed for distribution networks including forward-backward sweep method [9,10], compensation method [11], implicit Z-bus method [12-14], fuzzy logic method [15-17], and modified Newton or Newton like methods [18-21]. However, these methods depend on the convergence of iterative solutions to solve for unknowns in the algorithm. These methods can no longer be relied on when the convergence is not achieved, which is a challenge in LV networks.

Authors of [22] present a simplified load flow solution using active power and reactive power injections at a specified bus. However, this method is limited to an MV network and also requires iterative convergence techniques. In [23], the author presents a direct approach for unbalanced three-phase distribution load flow solutions which is robust and time-efficient. The approach proposed only requires the traditional bus-branch oriented data used by most DNSPs. However, the approach developed does not include a whole distribution network where transitions to different voltage levels happen.

The voltage variation across an entire distribution network can be easily understood through a thorough analytical formulation which includes both the MV and LV sides of the distribution network. This analytical formulation presents a distribution network without decoupling the LV network from the MV network, which is a necessity in order to construct a voltage control method that simultaneously regulates the voltage across both sides of the network. By exploring the network as a whole, the voltage control method can easily evaluate the influence of changes in one part of the network on other parts of the network. Hence, a new analytical formulation that presents the entire distribution network as one whole network is essential in the process of developing an effective volt/var control method. 
This paper presents an analytical formulation of a complete distribution network which covers the MV and LV sides of the network. The purpose of this analytical formulation is to be utilised in the development of an efficient volt/var control method. A generalised volt/var control method is developed using the analytical formulation, which is extended from [23] and also inherits the same advantages of it. The analytical formulation is applied on a modified IEEE 13 node test feeder which includes both MV and LV networks using a few scenarios of different PV system penetration. Results obtained from the proposed formulation are validated using the OpenDSS software. Also, the proposed volt/var control method is tested on the modified IEEE 13 node test feeder over a 24-hour period with varying loads and PV system penetration. Results obtained are compared with results using a conventional volt/var control method to verify its effectiveness.

The contributions of this paper include:

1. the presentation of a theoretical formulation for use in volt/var control method for combined MV and LV networks,

2. the inclusion of active and passive volt/var devices in MV and LV networks, and

3. the development of a holistic volt/var control method across MV and LV networks.

The rest of the paper is organised as follows. Section 2 presents the development of the proposed analytical formulation. Section 3 explains the development of the proposed generalised volt/var control method. Section 4 describes the network used for validation purposes. Section 5 presents results obtained and associated analysis from the proposed method and simulation. Section 6 concludes the paper and gives insight to future research directions.

\section{Analytical Formulation of a Holistic Distribution Network}

In this section, a new analytical formulation is developed considering a transition of different voltages, so that it is applicable to both sides of a distribution network. Fig. 1 shows an example of a generalised single-line diagram of a distribution network topology which consists of a zone substation (ZS), distribution substation (DS), three-phase distribution lines, loads across different phases, a capacitor bank and a PV system.

The proposed analytical formulation takes advantage of some of the basic features of a direct approach for a distribution network load flow solution proposed in [23]. The approach in [23] employs two different matrices and simple matrix multiplications to solve for voltage drops in a network. This approach starts with solving for branch currents based on all current injections in the network, which can represent a load or any voltage regulating devices in the network. Then, the voltage at a specific bus in the network can be found by solving the voltage drop between the reference bus and the specific bus, which is equivalent to the product of branch currents and branch impedances. The solution approach proposed in [23] is straightforward and only relies on the topological network data used by most utilities. With this approach, the LU decomposition and forward-backward substitution of the Jacobian matrix (or similar matrices) can be avoided, making it robust and efficient. This approach can also be used for load flow solution of weakly meshed distribution systems. This approach can be utilised on either the MV or LV sides of distribution 


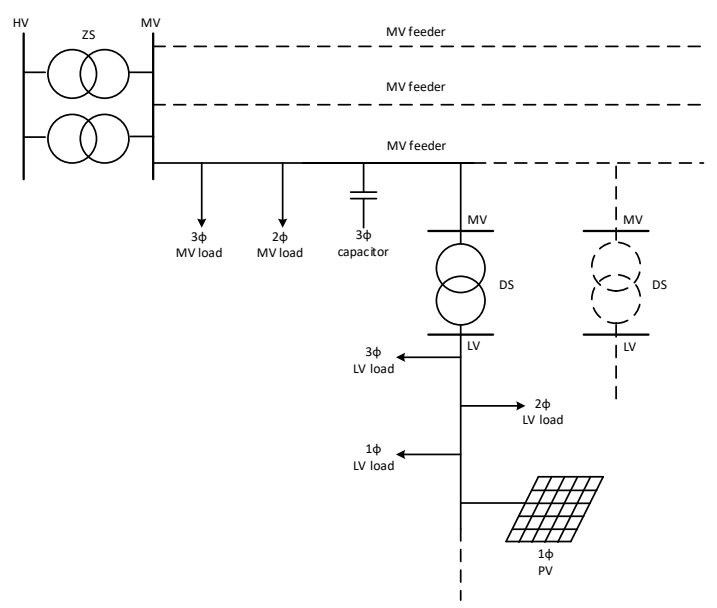

Figure 1: Generalised representation of a distribution network

networks, however it does not address the load flow solution across an entire distribution network, where there is a transition to different voltage levels.

Fig. 2 shows a per unit representation of a complete distribution network across one phase. Substation transformers and distribution lines are represented as impedances $(Z)$, while the load, capacitor and PV system are represented as positive or negative current injections, denoted by $I_{i, L}$, $I_{i, C}$ and $I_{i, P}$ respectively. Different types of loads such as constant current and constant impedance loads can be converted into current injections and included in the load flow as well. The conversion of distribution transformer depends on the winding and leakage impedance of the transformer. If phasors need to be taken into account for the transformer, different resistance and reactance values can be used to represent the phasors. Although not included in this paper, other voltage regulating devices such as voltage regulators, on load tap changers and energy storage systems can also be included as impedances and current injections.

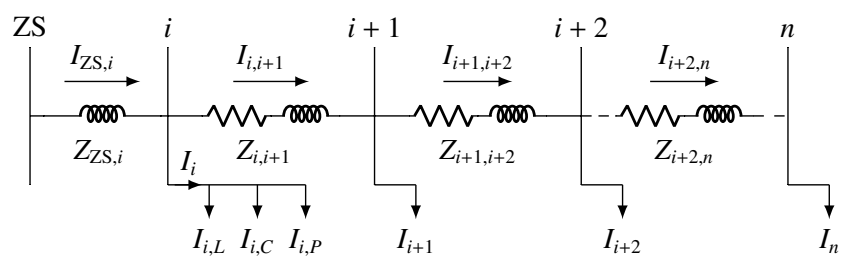

Figure 2: Per unit equivalent circuit for one phase across an MV and LV network

The distribution transformer, which is at a fixed tap, is the network element that separates the LV network from the MV network. The conversion of this transformer into an equivalent impedance bridges the gap between these two different voltage levels so that the network can now be treated as a single entity. Consequently, per unit representation of the network is imperative since its versatility allows it to function in different voltage levels at the same time. By undertaking such a conversion, the situation at the end of the LV network can be known from the ZS point of view and vice versa. The sensitivity of each buses to any changes in any part of the network can 
also be monitored easily. For example, the effect of PV systems in an LV network on MV buses at the other end of the network will be shown in Section 5. Knowledge of this information is crucial in the development of a voltage regulation method, and the ability to gain this information is aided with ease by this proposed formulation.

The base complex power, $S_{\text {base }}$, is equal to the kVA rating of the distribution transformer, while base voltages of MV and LV sides of the network are voltage ratings of the primary and secondary side of the transformer respectively. Although the single phase representation is shown below, same philosophy is undertaken for all three phases. Referring to Fig. 2, the complex load $S_{i, L}$ at bus $i$ is equivalent to:

$$
S_{i, L}=P_{i, L}+j Q_{i, L} \quad i=1 \ldots n
$$

where $P_{i, L}$ and $Q_{i, L}$ are the active and reactive load at bus $i$.

The equivalent load current at bus $i$ can be denoted by

$$
I_{i, L}^{k}=I_{i, L}^{\mathrm{re}}+j I_{i, L}^{\mathrm{im}}=\left(\frac{P_{i, L}+j Q_{i, L}}{V_{i}^{k}}\right)^{*}
$$

where $I_{i, L}^{\mathrm{re}}$ and $I_{i, L}^{\mathrm{im}}$ are real and imaginary components of the load current $I_{i, L}^{k}$ at the k-th iteration while $V_{i}^{k}$ is the bus voltage at the k-th iteration. For a voltage correction device that supplies active or reactive power (or operates at a leading power factor) such as capacitors and PV systems, the device is treated as a negative load. The current supplied by a capacitor, $I_{i, C}^{k}$, and a PV system, $I_{i, P}^{k}$, at bus $i$ at the k-th iteration are shown by (3) and (4) respectively.

$$
\begin{aligned}
& I_{i, C}^{k}=-I_{i, C}^{\mathrm{re}}-j I_{i, C}^{\mathrm{im}}=\left(\frac{-P_{i, C}-j Q_{i, C}}{V_{i}^{k}}\right)^{*} \\
& I_{i, P}^{k}=-I_{i, P}^{\mathrm{re}}-j I_{i, P}^{\mathrm{im}}=\left(\frac{-P_{i, P}-j Q_{i, P}}{V_{i}^{k}}\right)^{*}
\end{aligned}
$$

$I_{i, C}^{\mathrm{re}}$ and $I_{i, C}^{\mathrm{im}}$ are the real and imaginary components of the capacitor current while $Q_{i, C}$ is the equivalent var of the capacitor. Meanwhile, $I_{i, P}^{\mathrm{re}}$ and $I_{i, P}^{\mathrm{im}}$ are real and imaginary components of the PV system current and $P_{i, P}$ and $Q_{i, P}$ are the real and reactive power supplied by the PV system.

By applying Kirchhoff's Current Law (KCL), the total current injection at bus $i$ will be the sum of all currents present at the $\mathrm{k}$-th iteration.

$$
I_{i}^{k}=I_{i, L}^{k}+I_{i, C}^{k}+I_{i, P}^{k}
$$

By once again applying KCL, the branch current between two buses (for example the ZS bus and bus $i$ ) will be the sum of current injections from bus ZS to all downstream buses that are connected to it.

$$
I_{\mathrm{ZS}, i}^{k}=I_{i}^{k}+I_{i+1}^{k}+I_{i+2}^{k}+\ldots+I_{n}^{k}
$$




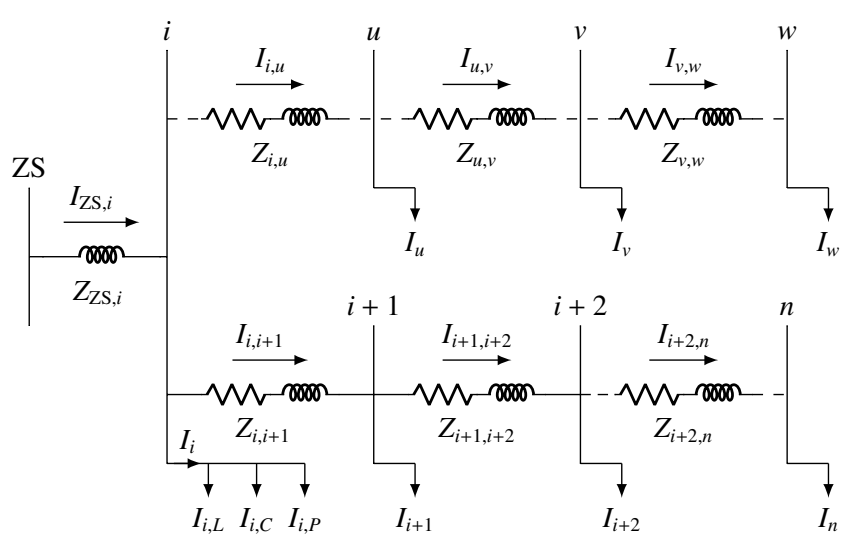

Figure 3: Per unit equivalent circuit with additional branches for one phase across an MV and LV network

The topology of realistic distribution networks are interconnected with multiple parallel branches in the MV and LV networks. Fig. 3 represents the generalised equivalent circuit when additional branches are present. Subsequently, $I_{\mathrm{ZS}, i}$ becomes:

$$
I_{\mathrm{ZS}, i}^{k^{\prime}}=I_{\mathrm{ZS}, i}^{k}+I_{u}^{k}+I_{v}^{k}+I_{w}^{k}
$$

By applying Kirchhoff's Voltage Law (KVL), the voltage drop, $\Delta V_{i}^{k}$, at the k-th iteration from the ZS to bus $i$ can be calculated as shown by (8):

$$
\Delta V_{i}^{k}=Z_{\mathrm{ZS}, i} I_{\mathrm{ZS}, i}^{k}
$$

where $Z_{\mathrm{ZS}, i}$ is the line impedance between the $\mathrm{ZS}$ and bus $i$. In the case where the network is unbalanced, the impedance in (8) will be replaced by a $3 \times 3$ matrix that includes self and mutual impedances from the line section while the current will be replaced by $3 \times 1$ matrix which contains the current for all three phases as shown by (9).

$$
\left(\begin{array}{l}
\Delta V_{a} \\
\Delta V_{b} \\
\Delta V_{c}
\end{array}\right)=\left(\begin{array}{lll}
Z_{a a} & Z_{a b} & Z_{a c} \\
Z_{b a} & Z_{b b} & Z_{b c} \\
Z_{c a} & Z_{c b} & Z_{c c}
\end{array}\right)\left(\begin{array}{c}
I_{a} \\
I_{b} \\
I_{c}
\end{array}\right)
$$

From (8) and (9), the bus voltage, $V_{i}$, can be found by subtracting $\Delta V_{i}^{k}$ from the ZS voltage, $V_{\mathrm{ZS}}$. From (6) and (8), $\Delta V_{i}$ can be expressed as:

$$
\begin{array}{r}
\Delta V_{i}=\left(R_{\mathrm{ZS}, i}+j X_{\mathrm{ZS}, i}\right)\left(I_{i, n}^{\mathrm{re}}+j I_{i, n}^{\mathrm{im}}\right) \\
\Delta V_{i}=R_{\mathrm{ZS}, i} I_{i, n}^{\mathrm{re}}-X_{\mathrm{ZS}, i} I_{i, n}^{\mathrm{im}}+j\left(X_{\mathrm{ZS}, i} I_{i, n}^{\mathrm{re}}+R_{\mathrm{ZS}, i} I_{i, n}^{\mathrm{im}}\right)
\end{array}
$$

where $R_{\mathrm{ZS}, i}$ and $X_{\mathrm{ZS}, i}$ are the line resistance and reactance between ZS bus and bus $i$ and $I_{i, n}^{\mathrm{re}}$ and $I_{i, n}^{\mathrm{im}}$ are the real and imaginary current injections from bus $i$ to bus $n$.

Similarly, with additional branches, from (7) and (8), $\Delta V_{i}$ will be: 


$$
\begin{array}{r}
\Delta V_{i}^{\prime}=\left(R_{\mathrm{ZS}, i}+j X_{\mathrm{ZS}, i}\right)\left(I_{i, n}^{\mathrm{re}}+I_{i, w}^{\mathrm{re}}+j I_{i, n}^{\mathrm{im}}+j I_{i, w}^{\mathrm{im}}\right) \\
\Delta V_{i}^{\prime}=R_{\mathrm{ZS}, i}\left(I_{i, n}^{\mathrm{re}}+I_{i, z}^{\mathrm{re}}\right)-X_{\mathrm{ZS}, i}\left(I_{i, n}^{\mathrm{im}}+I_{i, z}^{\mathrm{im}}\right)+j\left[X_{\mathrm{ZS}, i}\left(I_{i, n}^{\mathrm{re}}+I_{i, z}^{\mathrm{re}}\right)+R_{\mathrm{ZS}, i} I\left(I_{i, n}^{\mathrm{im}}+I_{i, z}^{\mathrm{im}}\right)\right]
\end{array}
$$

In general, the real and imaginary components of $\Delta V$ can be represented as:

$$
\begin{array}{r}
\Delta V^{\mathrm{re}}=I^{\mathrm{re}} R-I^{\mathrm{im}} X \\
\Delta V^{\mathrm{im}}=I^{\mathrm{re}} X+I^{\mathrm{im}} R .
\end{array}
$$

The formulation can be used to perform a sensitivity analysis to determine the factor which $V$ and $\Delta V$ are most dependant on. This is as the formulation demonstrates the continuity and spread of $\Delta V$ throughout the entire MV and LV networks. With this, the voltage requirement across the entire network is known and voltage control actions can be planned accordingly. For example, the level of reactive power required to regulate the voltage level measured at a bus can be determined by working out the corresponding current injection needed to increase or decrease $\Delta V$ at the bus. By knowing the exact requirement across the entire network, the consequent voltage control action taken will accommodate each bus regardless of its location in the network, and in addition, unnecessary voltage compensation actions can also be avoided.

\section{Devising a Volt/Var Control Method using the Proposed Theoretical Formulation}

Advantages of the proposed theoretical formulation are exploited to develop a volt/var control method that manage an entire distribution network. The theoretical formulation:

i) allows observation of the whole distribution network as a single, top-down network from the $\mathrm{ZS}$ to the end LV customer.

ii) solves branch currents, voltages and voltage drops at all nodes in the network.

iii) details all voltage control devices in the network (capacity, location, etc) and their sensitivity to variations in network voltages.

The main objectives of the proposed volt/var control method are to (i) ensure that voltage levels in the network are within established boundaries and statutory requirements, (ii) the minimum number of tap change operations are performed by the OLTC, and (iii) the maximum active power injection from PV systems can be achieved. There are several steps associated with the generalised volt/var control method to ensure that these objectives are met. Before volt/var control is established for the network, a lookup table which contains the sensitivity of control devices in the network requires development. The lookup table will be used in the proposed volt/var control method to decide on appropriate control actions. 


\subsection{Development of a Lookup Table}

In this paper, volt/var control devices taken into account are capacitors and PV systems, however, other devices can also be included in the lookup table. When a capacitor is connected to a network, the respective current supplied by the capacitor is calculated using (3). From (10), the new $\Delta V, \Delta V_{i, C}$, is therefore:

$$
\Delta V_{i, C}=\left(I_{i, n}^{\mathrm{re}}+j I_{i, n}^{\mathrm{im}}+j I_{i, C}^{\mathrm{im}}\right)\left(R_{\mathrm{ZS}, i}+j X_{\mathrm{ZS}, i}\right)
$$

Similarly, when a PV system is connected, $\Delta V_{i, P}$ can be expressed as:

$$
\Delta V_{i, P}=\left(I_{i, n}^{\mathrm{re}}+j I_{i, n}^{\mathrm{im}}+I_{i, P}^{\mathrm{re}}+j I_{i, P}^{\mathrm{im}}\right)\left(R_{\mathrm{ZS}, i}+j X_{\mathrm{ZS}, i}\right)
$$

Assuming that the network operating condition remains the same, the corresponding improvements of $\Delta V$ at bus $i$ as a result of the capacitor operation, $\Delta V_{\mathrm{imp}, C}$, or PV system operation, $\Delta V_{\mathrm{imp}, P}$, are calculated as follow:

$$
\begin{array}{r}
\Delta V_{\mathrm{imp}, C}=\Delta V_{i, C}-\Delta V_{i} \\
\Delta V_{\mathrm{imp}, P}=\Delta V_{i, P}-\Delta V_{i} \\
\Delta V_{\mathrm{imp}, C}=\left(j I_{i, C}^{\mathrm{im}}\right)\left(R_{\mathrm{ZS}, \mathrm{PCC}}+j X_{\mathrm{ZS}, \mathrm{PCC}}\right) \\
\Delta V_{\mathrm{imp}, P}=\left(I_{i, P}^{\mathrm{re}}+j I_{i, P}^{\mathrm{im}}\right)\left(R_{\mathrm{ZS}, \mathrm{PCC}}+j X_{\mathrm{ZS}, \mathrm{PCC}}\right)
\end{array}
$$

where $R_{\mathrm{ZS}, \mathrm{PCC}}$ and $j X_{\mathrm{ZS}, \mathrm{PCC}}$ are the line resistance and reactance from the ZS to the point of common connection (PCC) between bus $i$ and the bus where the control device is connected.

For a capacitor, only $j I_{i, C}^{\mathrm{im}}$ is taken into account under the assumption that minimal real current component is injected. In this paper, all PV systems are operating at unity power factor, therefore only $I_{i, P}^{\mathrm{re}}$ is calculated, assuming that minimal $I_{i, P}^{\mathrm{im}}$ is injected. Therefore, $\Delta V_{\mathrm{imp}, P}$ can be simplified as:

$$
\Delta V_{\mathrm{imp}, P}=\left(I_{i, P}^{\mathrm{re}}\right)\left(R_{\mathrm{ZS}, \mathrm{PCC}}+j X_{\mathrm{ZS}, \mathrm{PCC}}\right)
$$

The magnitude of voltage improvement, $\left|\Delta V_{\text {imp }}\right|$, at different buses as a result of different control device operation is listed in a lookup table. This process is repeated for each control device in the network until the lookup table is completed.

Table 1 shows an example of the lookup table used in this paper wherein entries correspond to the number of control devices and buses in the network.

\subsection{Proposed Generalised Volt/Var Control Method}

This section describes the benefits of the proposed volt/var control on combined MV-LV networks and the implementation of the proposed volt/var control in distribution networks. 
Table 1: Generalised lookup table

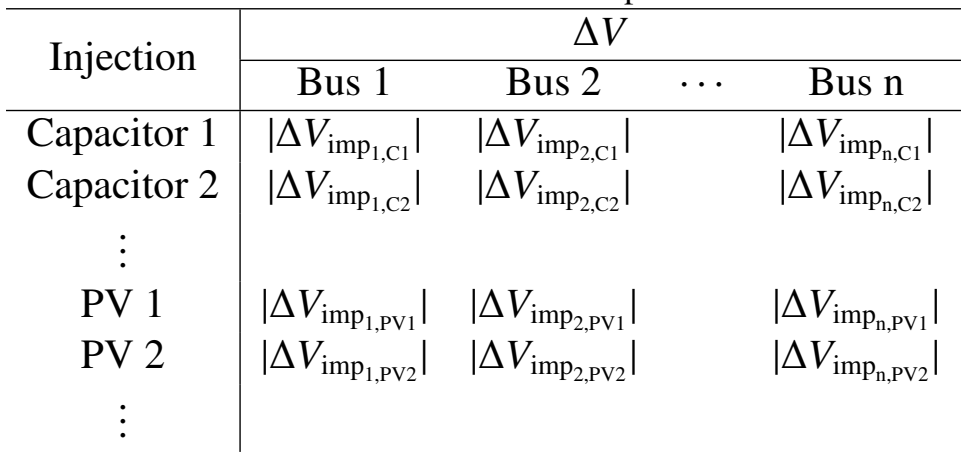

\subsubsection{Motivation of the proposed volt/var control method}

Using the proposed volt/var control method, the realistic topology of interconnected MV and LV networks with unbalanced distribution of loads and PV systems are taken into account. This ensures that the average three-phase as well the individual phase voltage levels across the entire network are within stipulated limits.

The segregation of voltage control strategies in MV and LV networks as a common practice may no longer be adequate in currently evolving distribution networks. When there is no coordination between control devices in a distribution network, redundant or conflicting control operations may be carried out by multiple devices in the network. The proposed volt/var control method eliminate the risk of excessive control device operations, ensuring an optimal network performance.

When compared to other volt/var control methods $[4,6,7]$, the volt/var control actions undertaken by the proposed control method are based on the state of both MV and LV networks. Therefore, any control actions that may deteriorate the voltage on either sides of the network can be avoided. If there is a viable control action on one side of the network that can improve the voltage magnitude on the other side of the network, this will also be taken into consideration. The proposed volt/var control method also prioritises control actions that will globally improve the voltage magnitude across the network with minimal device operations as will be demonstrated in Sections 5.2 and 5.3.

High PV system penetration in LV networks can cause violation of steady state voltage level, not only in LV networks but can also cause reverse power flow into MV networks [8]. The proposed volt/var control can accommodate more PV system penetration by utilising other available control devices in both MV and LV networks to improve the voltage level across the entire network, thereby avoiding active power curtailment.

\subsubsection{Implementation of the proposed volt/var control method}

Under the proposed volt/var control method, the voltage magnitude across the entire network at each phase is monitored at every 15 minutes to prevent any voltage limit violations, as shown by (17). In this case, only the voltage magnitude is taken into account since the voltage phase angle is not bounded with the established standard.

$$
\left|V_{\text {minimum }}\right| \leq\left|V_{\text {warn-min }}\right| \leq\left|V_{i}\right| \leq\left|V_{\text {warn-max }}\right| \leq\left|V_{\text {maximum }}\right|
$$


The minimum and maximum voltage limits can be the standardised allowable voltage range establised by an Australian Standard, for example, or a smaller range to ensure that the voltage level in the network will never stray outside prescribed limits. The limits can also be different for any buses in the network, for instance, different set of limits can be used in the MV and LV networks as they may have different voltage requirements.

If a voltage level violates the preset maximum and minimum ranges, an emergency signal will be sent to the control system, so that an immediate action can be taken. In addition, the 'health' of voltage levels across the entire network is graded. When a voltage level is within $V_{\text {warn-min }}$ and the minimum limit or $V_{\text {warn-max }}$ and the maximum limit, a warning signal will be sent to the control system. This is to ensure that any correcting action will not exacerbate the voltage level in the warning range.

When a voltage limit is violated, the control system will calculate the voltage magnitude required to bring the voltage back within safe limits as shown by (18).

$$
\left|\Delta V_{\text {required }}\right|=\left|V_{\text {ideal }}\right|-\left|V_{i}\right|
$$

$\left|V_{\text {ideal }}\right|$ used in (18) is between $V_{\text {warn-min }}$ and $V_{\text {warn-max }}$ to ensure that $\left|V_{i}\right|$ improves significantly and remains close to the nominal value. A negative $\left|\Delta V_{\text {required }}\right|$ indicates that the voltage at the bus must be reduced while a positive $\left|\Delta V_{\text {required }}\right|$ indicates that the voltage must be increased.

The lookup table is updated everytime a voltage violation happens in the network, as the system condition changes all the time with changing loads and varying control actions. $\left|\Delta V_{\text {required }}\right|$ is then matched with $\left|\Delta V_{\text {imp }_{i}}\right|$ at the respective bus in the lookup table based on several hierarchical conditions that will ensure:

i. the voltage level across the entire network be within prescribed limits,

ii. the maximum active power is injected through PV systems,

iii. the minimum number of tap change operations are performed by the OLTC at the ZS, and

iv. the number of consecutive volt/var device operations is minimised.

The control actions requested by the proposed volt/var control method are intended to accomplish the set hierarchical conditions based on the availability of control devices. The first condition will ensure that each warning signal sent to the control system is taken into account before making any voltage correction action. In case where several voltage violations happen at the same time, the control system will check for redundant control action that can be used as a holistic volt/var control action instead of a localised one. For example, when two buses from different feeders require a voltage boost, the control system will decide on a single holistic action that will satisfy the voltage requirement of both buses instead of two separate localised control actions.

In order to avoid redundant or conflicting device operations in the network, the fourth condition is included as a separate measure from the normal device hysteresis. However, in case where conflicting voltage violations happen at the same time, simultaneous or consecutive device operations will be allowed to ensure that all voltage level across the network remain within limits. 


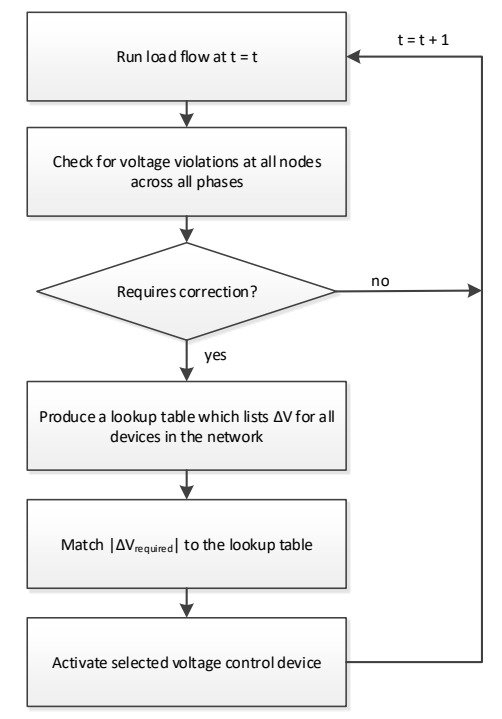

Figure 4: Flowchart of the proposed volt/var control method

Fig. 4 summarises the proposed generalised volt/var control method. The proposed volt/var control method takes advantage of the holistic view across an entire distribution network to ensure that voltage regulation is performed efficiently on the network. Since network voltage levels and the status of control devices across the network are inputs to the control system, unwanted and unnecessary control actions can be avoided. The hierarchical conditions of the proposed volt/var control method can be modified depending on criteria that are most important to a DNSP. Therefore, the volt/var control method offers the choice of being selective on which control devices to utilise. As the proposed control does not include optimisation algorithms, it is can be feasibly adopted by DNSPs for realistic networks.

\section{Network Model}

The extended analytical formulation and the proposed volt/var control method is applied on a modified IEEE 13 node test feeder to verify its effectiveness. The original IEEE 13 node test feeder consists of an MV network and only an aggregated LV load on the secondary side of a distribution substation. In order to demonstrate the veracity of the proposed formulation, a distribution network which consists of both MV and LV networks is required. This network can also be used in the future as a platform to develop a voltage regulation method across an entire distribution network. The IEEE 13 node test feeder has been extended to include a portion of LV network which is inspired by a typical residential area in a semi-rural part of Australia. Figs. 5 and 6 show the MV and LV networks of the modified IEEE 13 node test feeder respectively. The LV network is extended from node 634, where there are two LV feeders connected to the distribution substation rated at $500 \mathrm{kVA}$, supplying 31 single-phase residential customers. All customers have a singlephase rooftop PV system rated at $10 \mathrm{kVA}$ for phases $\mathrm{A}$ and $\mathrm{C}$ and $15 \mathrm{kVA}$ for phase $\mathrm{B}$. The ratings of these PV systems are specified to be higher than the allowable rating for PV systems in 


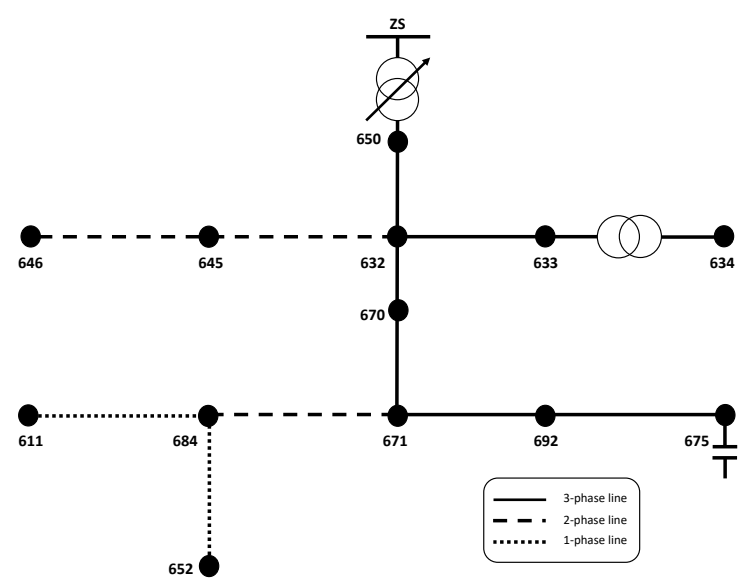

Figure 5: Modified IEEE 13-node test feeder [24]

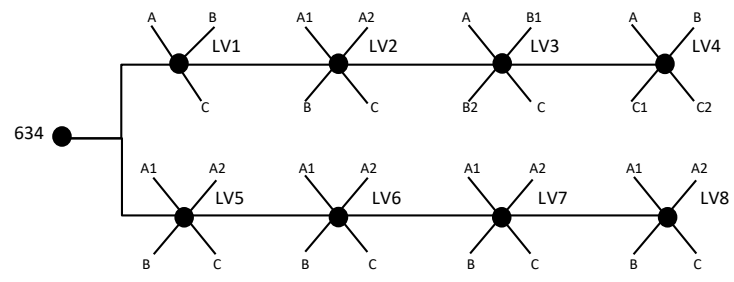

Figure 6: LV network extension

Australian standard AS4777.1 to demonstrate the effect of growing PV penetration in distribution networks [25]. All PV systems are operated at a unity power factor.

In the modified IEEE 13 node test feeder, the voltage regulator between nodes 650 and 632 and the single-phase capacitor at the node 611 are removed. The transformer at the ZS is modelled with an OLTC. The 600 kvar capacitor bank at node 675 is modelled as a switched capacitor with 150 kvar per step. Node 670 in Fig. 5 is one-third of the distance between nodes 632 and 671, where the distributed load is connected. The loading on the MV network follows the loading of IEEE 13 node test feeder [24] and the load on the LV network is distributed across the three phases as shown in Table 2.

Each line section between LV nodes is a three-phase, 80 feet line, while each of the line sections supplying the load from the PCC is a single-phase, 35 feet line. Tables 3 and 4 show line impedances for the three-phase line and the single-phase line respectively.

The minimum and maximum voltage limits in (17) for this test feeder are specified to be $-5 \%$ and $+9 \%$ for the LV network side and $\pm 9 \%$ for the MV network side which is slightly stricter than the standard Australian voltage range [26]. This is to ensure that voltage correction actions take place before the voltage level actually violates statutory limits. $V_{\text {warn-min }}$ and $V_{\text {warn-max }}$ for LV network side are $-4 \%$ and $+8 \%$ respectively, while $V_{\text {warn-min }}$ and $V_{\text {warn-max }}$ for MV network side are $\pm 8 \%$. 
Table 2: LV network load data

\begin{tabular}{|c|c|c|c|}
\hline \multirow{2}{*}{ Node } & \multicolumn{3}{|c|}{$\operatorname{Load}(\mathrm{kVA})$} \\
\hline & Phase A & Phase B & Phase C \\
\hline LV1 & $11+\mathrm{j} 4$ & $11+j 4$ & $11+\mathrm{j} 4$ \\
\hline \multirow{2}{*}{ LV2 } & A1: $13+j 6$ & \multirow{2}{*}{$11+j 4$} & \multirow{2}{*}{$11+j 4$} \\
\hline & A2: $11+j 4$ & & \\
\hline \multirow{2}{*}{ LV3 } & \multirow{2}{*}{$13+j 6$} & $B 1: 10+j 3$ & \multirow{2}{*}{$11+j 4$} \\
\hline & & $B 2: 10+j 3$ & \\
\hline \multirow{2}{*}{ LV4 } & \multirow{2}{*}{$11+\mathrm{j} 4$} & \multirow{2}{*}{$13+j 3$} & $13+j 6$ \\
\hline & & & $13+j 6$ \\
\hline \multirow{2}{*}{ LV5 } & A1: $13 \mathrm{j} 6$ & \multirow{2}{*}{$10+j 3$} & \multirow{2}{*}{$13+j 6$} \\
\hline & A2: $15+\mathrm{j} 10$ & & \\
\hline \multirow{2}{*}{ LV6 } & A1: $11+\mathrm{j} 4$ & \multirow{2}{*}{$10+j 3$} & \multirow{2}{*}{$11+\mathrm{j} 4$} \\
\hline & A2: $11+\mathrm{j} 4$ & & \\
\hline \multirow{2}{*}{ LV7 } & A1: $11+\mathrm{j} 4$ & \multirow{2}{*}{$10+j 3$} & \multirow{2}{*}{$11+j 4$} \\
\hline & A2: $13+j 6$ & & \\
\hline \multirow{2}{*}{ LV8 } & A1: $11+j 4$ & \multirow{2}{*}{$10+j 3$} & \multirow{2}{*}{$11+j 4$} \\
\hline & A2: $11+\mathrm{j} 4$ & & \\
\hline
\end{tabular}

Table 3: Three-phase line impedance

\begin{tabular}{|c|c|c|c|c|c|}
\hline \multirow{2}{*}{ Length } & \multicolumn{2}{|c|}{$\mathrm{R}(\Omega / \mathrm{km})$} & \multicolumn{2}{c|}{$\mathrm{X}(\Omega / \mathrm{km})$} & \multirow{2}{*}{$\mathrm{C}(\mathrm{nF} / \mathrm{km})$} \\
\cline { 2 - 5 } & Self & Mutual & Self & Mutual & \\
\hline 80 & 0.4827 & 0.1607 & 0.0803 & 0.0063 & 0 \\
\hline
\end{tabular}

Table 4: Single-phase line impedance

\begin{tabular}{|c|c|c|c|}
\hline Length(ft) & $\mathrm{R}(\Omega / \mathrm{kft})$ & $\mathrm{X}(\Omega / \mathrm{kft})$ & $\mathrm{C}(\mathrm{nF} / \mathrm{kft})$ \\
\hline 35 & 0.25 & 0.076 & 3 \\
\hline
\end{tabular}




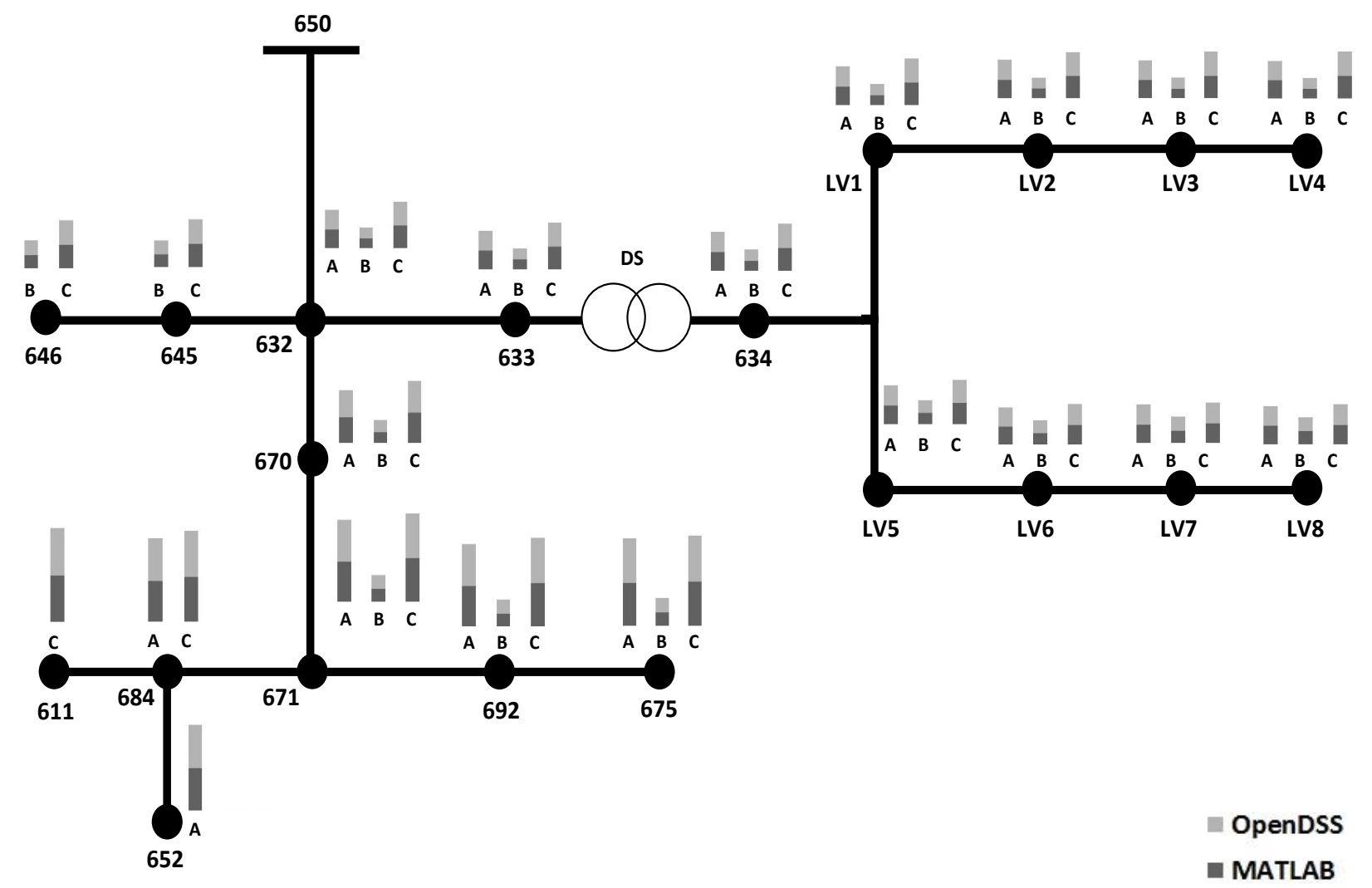

Figure 7: $|\Delta V|$ across the modified IEEE 13 node test feeder including LV network extension

\section{Results and Analyses}

\subsection{Verification of the Proposed Theoretical Formulation}

The proposed analytical formulation is solved in MATLAB and results obtained are compared with a load flow solution from OpenDSS. To analyse the accuracy of the proposed formulation under different network conditions, four different scenarios are tested, which are:

1. Peak loads with no PV system penetration.

2. Peak loads with peak PV system penetration.

3. Peak MV loads, $40 \%$ of peak LV loads and $40 \%$ of peak PV system penetration.

4. Peak MV loads, $40 \%$ of peak LV loads and peak PV system penetration.

Fig. 7 presents magnitudes of $\Delta V$ across the modified IEEE 13 node test feeder under case four using both the proposed formulation in MATLAB and simulation in OpenDSS. $|\Delta V|$ is from the node 650 which is the $\mathrm{ZS}$ and depends on which phase is available on the line sections. As seen from Fig. 7, $|\Delta V|$ obtained using the proposed formulation and simulation match, verifying the veracity of the proposed formulation. $|\Delta V|$ on phase $\mathrm{B}$ can be seen to be significantly lower 
Table 5: Summary of Results

\begin{tabular}{|l|l|c|c|}
\hline \multirow{2}{*}{ Case } & \multirow{2}{*}{ Phase } & \multicolumn{2}{|c|}{ Range of \% Error of Delta V } \\
\cline { 3 - 4 } & & Real & Imaginary \\
\hline \multirow{3}{*}{ Case 1 } & A & $0.005-0.403$ & $0.004-2.104$ \\
\cline { 2 - 4 } & $\mathrm{B}$ & $0.004-0.139$ & $0.393-2.419$ \\
\cline { 2 - 4 } & $\mathrm{C}$ & $0.000-0.331$ & $0.027-3.445$ \\
\hline \multirow{3}{*}{ Case 2 } & $\mathrm{A}$ & $0.004-0.368$ & $0.022-2.419$ \\
\cline { 2 - 4 } & $\mathrm{B}$ & $0.005-0.139$ & $0.231-2.737$ \\
\cline { 2 - 4 } & $\mathrm{C}$ & $0.000-0.313$ & $0.079-3.248$ \\
\hline \multirow{3}{*}{ Case 3 } & $\mathrm{A}$ & $0.000-0.418$ & $0.073-2.522$ \\
\cline { 2 - 4 } & $\mathrm{B}$ & $0.004-0.534$ & $0.268-3.320$ \\
\cline { 2 - 4 } & $\mathrm{C}$ & $0.003-0.278$ & $0.007-3.044$ \\
\hline \multirow{3}{*}{ Case 4 } & $\mathrm{A}$ & $0.007-0.422$ & $0.028-3.782$ \\
\cline { 2 - 4 } & $\mathrm{B}$ & $0.007-0.296$ & $0.085-3.569$ \\
\cline { 2 - 4 } & $\mathrm{C}$ & $0.003-0.318$ & $0.118-3.166$ \\
\hline
\end{tabular}

compared to that on phases $\mathrm{A}$ and $\mathrm{C}$ as the loading on phase $\mathrm{B}$ is considerably smaller than the loading on phases $\mathrm{A}$ and $\mathrm{C}$. As seen in Fig. 7, $|\Delta V|$ obtained using the theoretical formulation match that using OpenDSS.

The percentage error in $\Delta V$ is calculated as shown in (19). Table 5 summarises the range of percentage error for $\Delta V$ at all buses across the whole network obtained from the analytical formulation compared to the simulation results.

$$
\% \text { error }=\frac{\left|\Delta V_{\text {calculation }}-\Delta V_{\text {simulation }}\right|}{\Delta V_{\text {simulation }}}
$$

As seen, the error ranges from $0 \%$ to $3.8 \%$ for calculating $\Delta V$ at the third decimal point. This demonstrates that the error is negligible.

Based on Fig. 7, $|\Delta V|$ is shown to be dependant on the combination of net power flow and the impedance of line sections. The proposed theoretical formulation can be used to further analyse factors that affect $\Delta V$, and this knowledge can be used to determine the corresponding voltage control actions required to regulate the voltage level in distribution networks.

\subsection{Implementation of the Proposed Volt/Var Control Method}

The modified IEEE 13 node test feeder is modelled in OpenDSS while the volt/var control method is executed from MATLAB. OpenDSS runs the load flow and passes voltage, current and power levels and control device status to the control algorithm in MATLAB where the processing is done to determine any necessary control actions. Then MATLAB sends the appropriate command back to OpenDSS. The load flow runs over a 24-hour period with a varying load profile for every 15-minute intervals, starting at midnight. The control device sensitivity lookup table is produced separately before the load flow is run. For the test network, entries of the lookup table include all steps of the switched capacitor bank and all PVs in the LV network. The voltage at the ZS is set initally to 1.06 p.u. while the capacitor bank at the node 675 is disconnected from the network. 


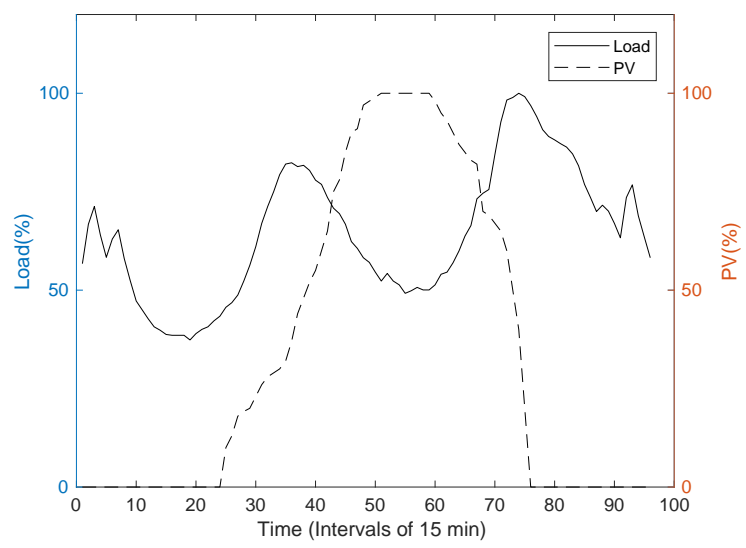

Figure 8: Load profile and PV profile

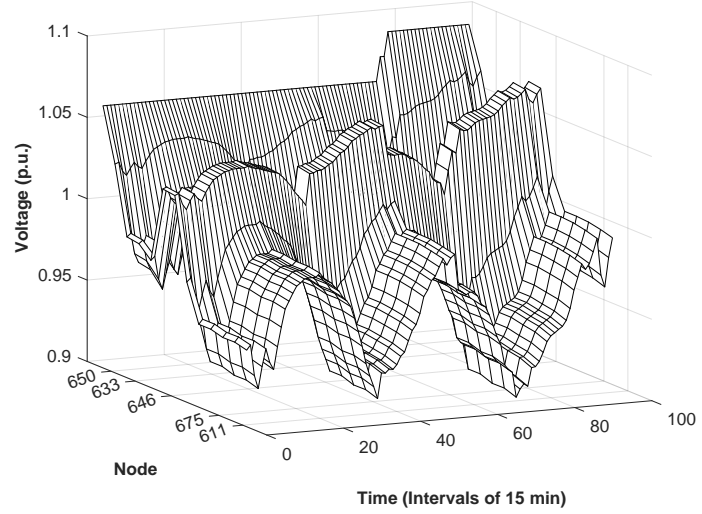

Figure 9: Voltage profile using proposed control for the MV network at phase C

Fig. 8 shows the varying load profile and the PV profile across the 24-hour period. The load profile is obtained from a typical semi-rural distribution network in New South Wales, Australia while the PV profile is a typical active power output by a PV system on a sunny day. The load profile shown is in percentage of the original peak loading condition while the PV profile is in percentage of the PV inverter rating.

Using the proposed volt/var control method, the voltage level across the network remains within allowable ranges throughout the 24-hour period. The voltage level on phase B is consistently the highest among three phases while the voltage level on phase $\mathrm{C}$ is consistently the lowest among three phases due to the unbalanced loading in the network. The volt/var control algorithm acts according to emergency signals sent from phases $\mathrm{B}$ and $\mathrm{C}$, consequently improving the voltage level across all three phases. Fig. 9 shows the voltage profile across the MV network at phase $\mathrm{C}$, where the voltage level is the lowest among all three phases while Fig. 10 shows the voltage profile across the LV network at phase $\mathrm{B}$, where the voltage level is the highest among all three phases. As seen from Fig. 9, at the 35th interval, the voltage at the node 611 drops below 0.91 p.u., triggering the emergency signal. The volt/var control algorithm matches the required $\Delta V$ 


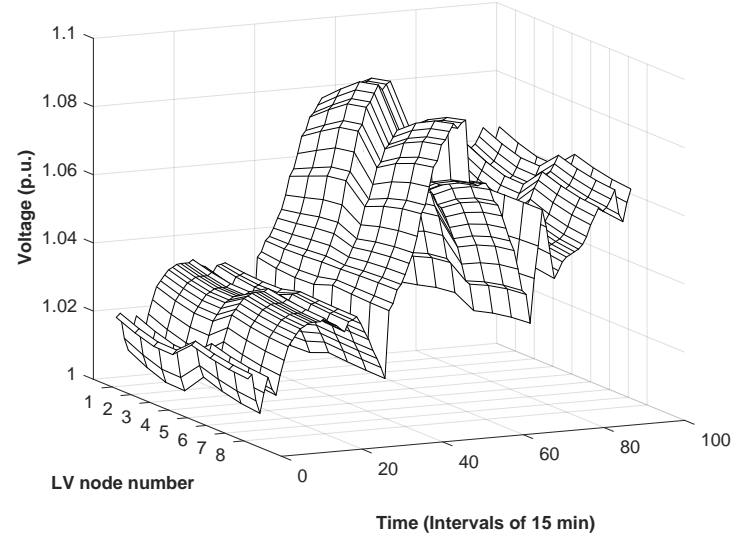

Figure 10: Voltage profile using proposed control for the LV network at phase B

improvement to the lookup table and selects a capacitor injection of $600 \mathrm{kVar}$ into the network, raising the voltage level to within the required limit.

From Fig. 10, at the 55th interval with maximum PV penetration, the voltage level at the node LV4 rises to 1.091 p.u. From the lookup table, the operation of disconnecting the $600 \mathrm{kvar}$ capacitor bank at the node 675 in the MV network is an ideal option. With this operation, all PV inverters in the LV network can continue injecting active power while the OLTC operation can be avoided. This highlights the importance of a holistic volt/var control action which ensures that voltage levels across the entire distribution network are within limits while PV inverters are operating at their full potential.

From Figs. 8 and 9 at the 70th interval, as the load increases, the minimum voltage recorded drops to 0.9 p.u. at the node 611 , resulting in a 600 kvar capacitor swithing in to the network. However, as the load continues to increase, two subsequent tap change operations are required to bring the voltage level within allowable limits.

In total, volt/var correction actions using the proposed method throughout the 24-hour period include three capacitor switchings and two tap change operations. Additionally, all PV systems are allowed to inject maximum active power during the 24-hour period. The network however shows voltage unbalance level of 3.5\% during the 24-hour period when irradiance is available. The proposed volt/var control method can be extended to include a more sophisticated control of different PV inverter operations. An example of the application of different PV inverter operations to improve voltage regulation while reducing voltage unbalance at the same time is presented in [27].

\subsection{Comparison of the Proposed Volt/Var Control Method with the Conventional Volt/Var Method}

To verify the effectiveness of the proposed volt/var control method, the results are compared to the results obtained using a conventional volt/var control where in the MV network, the OLTC operates separately from the capacitor bank and do not regulate the voltage in the LV network. As generally adopted by DNSPs, the OLTC conventionally operates by changing the tap position when the voltage level at the secondary side goes out of the allowed bandwidth, while the capacitor 


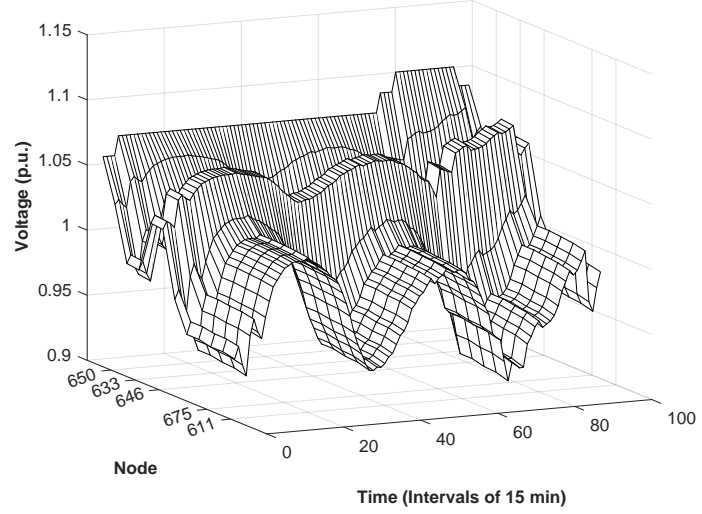

Figure 11: Voltage profile using conventional method for the MV network at phase C

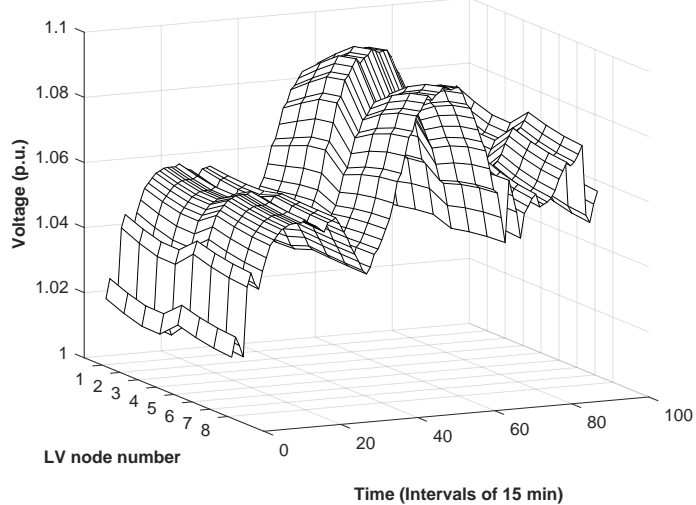

Figure 12: Voltage profile using conventional method for the LV network at phase B 


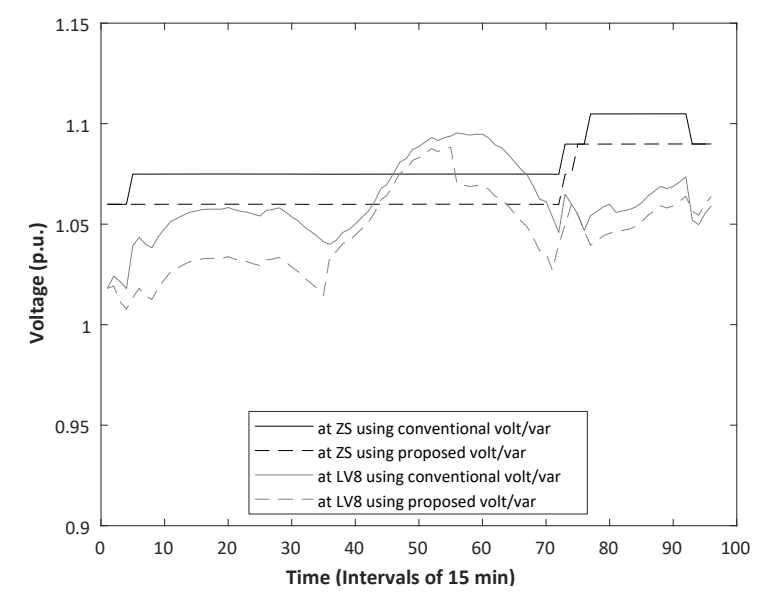

Figure 13: Comparative analysis between the proposed and the conventional volt/var control methods

bank operates when the reactive power measurement at its location goes outside specified limits. In this case, PV systems in the LV network do not participate in voltage regulation.

Using the conventional method, the voltage level on phase B is consistently the highest among three phases while the voltage level on phase $\mathrm{C}$ is consistently the lowest among three phases throughout the simulation period, similar to the proposed volt/var control method. Fig. 11 shows the voltage profile on phase $\mathrm{C}$ across the MV network while Fig. 12 shows the voltage profile at phase $\mathrm{B}$ across the $\mathrm{LV}$ network using the conventional volt/var control method. As seen from Fig. 11, everytime the voltage level becomes closer to the maximum or minimum allowable limits, the OLTC operates, bringing the voltage closer to 1.0 p.u. At the 76th interval, due to the high load, the OLTC changes the tap position to give maximum voltage of 1.1 p.u.

The capacitor bank operates independently from the OLTC, based on the kvar measurement at the point of capacitor connection. At the 1 st interval, the capacitor switches on $300 \mathrm{kVar}$ to reduce the lagging reactive power. When the load increases at the 72nd interval, the capacitor switches on the remaining capacity to improve the voltage profile. As the load decreases during the 80th interval, the capacitor switches off a step to provide only $450 \mathrm{kVar}$ of reactive power.

Since PV systems do not participate in voltage regulation, they are allowed to inject as much active power as generated during the day. As seen from Fig. 12, the maximum voltage recorded is 1.098 p.u., which is very close to the maximum allowable magnitude. In total, the number of tap changes during the 24-hour period is four, with additional three capacitor switchings.

The voltage levels at the ZS (MV network) and node LV8 are close to the stipulated limits and prone to voltage violations. Accordingly, the comparative analysis between the proposed volt/var control method and conventional volt/var control method at the ZS and node LV8 for a typical day is depicted in Fig. 13. As seen, Fig. 13 highlights improvements in the network performance as a result of the proposed volt/var control method. Using the conventional method, in addition to voltage violations in both MV and LV networks, the total number of control actions are more than that of using the proposed volt/var control. This shows that independent consideration of MV and LV networks will limit the implementation of volt/var control methods at local levels. Under the proposed volt/var control method however, there is an improvement in voltage profile across the 
entire network with significantly less number of control actions.

\section{Conclusions}

This paper proposes a solution for voltage regulation in a combined MV and LV network using an integrated volt/var control method. First, a direct and efficient theoretical formulation is developed to solve the load flow of a complete MV-LV distribution network with minimal data related to the network topology and current injections. The proposed theoretical formulation is versatile as it can include any type of voltage regulating devices in an interconnected network as impedances and/or current injections. The proposed formulation is tested on a modified IEEE 13 node test feeder which includes an MV network and a section of an LV network. The results obtained from the proposed formulation correspond to the results obtained using simulation from OpenDSS with minimal computational errors. The proposed formulation is further used as an analytical tool for the development of voltage regulation strategy by devising the volt/var control method for an integrated MV-LV distribution network. The proposed volt/var control method regulates the tested distribution network effectively while ensuring maximum active power injection through PVs. Comparative analysis between the proposed volt/var control method with the conventional volt/var control method verifies that the proposed volt/var control method can improve the voltage profile of the tested MV-LV distribution network with minimum number of control operations.

\section{References}

[1] Harlow J. Electric Power Transformer Engineering (3rd Edition). CRC Press; 2012. ISBN 978-1-4398-5629-1.

[2] Paaso EA, Liao Y, Cramer AM. Formulation and solution of distribution system voltage and var control with distributed generation as a mixed integer non-linear programming problem. Electric Power Systems Research 2014;108:164 -9. doi:https://doi.org/10.1016/j.epsr.2013.11.016.

[3] Homaee O, Zakariazadeh A, Jadid S. Real-time voltage control algorithm with switched capacitors in smart distribution system in presence of renewable generations. International Journal of Electrical Power \& Energy Systems 2014;54:187 -97. doi:https://doi.org/10.1016/j.ijepes.2013.07.010.

[4] Afandi I, Ciufo P, Agalgaonkar A, Perera S. A comparison of voltage regulation and control methods. In: Power Engineering Conference (AUPEC), 2015 Australasian Universities. 2015, p. 1-6. doi: 10.1109/AUPEC.2015.7324878.

[5] Juamperez M, Yang G, Kjær S. Voltage regulation in LV grids by coordinated volt-var control strategies. Journal of Modern Power Systems and Clean Energy 2014;2(4):319-28. doi:10.1007/s40565-014-0072-0.

[6] Long C, Ochoa LF. Voltage Control of PV-Rich LV Networks: OLTC-Fitted Transformer and Capacitor Banks. IEEE Transactions on Power Systems 2015;PP(99):1-10. doi:10.1109/TPWRS.2015.2494627.

[7] Efkarpidis N, Rybel TD, Driesen J. Voltage regulation strategies for Flemish LV distribution grids utilizing single-phase PV inverters. In: 2015 IEEE 6th International Symposium on Power Electronics for Distributed Generation Systems (PEDG). 2015, p. 1-8. doi:10.1109/PEDG.2015.7223097.

[8] Walling RA, Saint R, Dugan RC, Burke J, Kojovic LA. Summary of distributed resources impact on power delivery systems. IEEE Transactions on Power Delivery 2008;23(3):1636-44. doi:10.1109/TPWRD.2007.909115.

[9] Kersting WH. Distribution System Modeling and Analysis, Third Edition. CRC Press; 2012. ISBN 9781439856222.

[10] Ghatak U, Mukherjee V. An improved load flow technique based on load current injection for modern distribution system. International Journal of Electrical Power \& Energy Systems 2017;84:168 -81. doi: https://doi.org/10.1016/j.ijepes.2016.05.008. 
[11] Shirmohammadi D, Hong HW, Semlyen A, Luo GX. A compensation-based power flow method for weakly meshed distribution and transmission networks. IEEE Transactions on Power Systems 1988;3(2):753-62. doi: $10.1109 / 59.192932$.

[12] Chen TH, Chen MS, Hwang KJ, Kotas P, Chebli EA. Distribution system power flow analysis-a rigid approach. IEEE Transactions on Power Delivery 1991;6(3):1146-52. doi:10.1109/61.85860.

[13] Farag HE, El-Saadany E, Shatshat RE, Zidan A. A generalized power flow analysis for distribution systems with high penetration of distributed generation. Electric Power Systems Research 2011;81(7):1499 -506. doi: https://doi.org/10.1016/j.epsr.2011.03.001.

[14] Hooshyar H, Vanfretti L. Multiphase unbalanced power flow and fault analysis of distribution networks with high penetration of inverter-interfaced ders. In: Applied Electrical Engineering and Computing Technologies (AEECT), 2015 IEEE Jordan Conference on. 2015, p. 1-8. doi:10.1109/AEECT.2015.7360585.

[15] Vlachogiannis JG. Fuzzy logic application in load flow studies. IEE Proceedings - Generation, Transmission and Distribution 2001;148(1):34-40. doi:10.1049/ip-gtd:20010032.

[16] Liu Y, Zhang P, Qiu X. Optimal volt/var control in distribution systems. International Journal of Electrical Power \& Energy Systems 2002;24(4):271-6.

[17] Bijwe PR, Raju GKV. Fuzzy distribution power flow for weakly meshed systems. IEEE Transactions on Power Systems 2006;21(4):1645-52. doi:10.1109/TPWRS.2006.881138.

[18] Nguyen HL. Newton-raphson method in complex form [power system load flow analysis]. IEEE Transactions on Power Systems 1997;12(3):1355-9. doi:10.1109/59.630481.

[19] Yang NC, Chen HC. Decomposed newton algorithm-based three-phase power-flow for unbalanced radial distribution networks with distributed energy resources and electric vehicle demands. International Journal of Electrical Power \& Energy Systems 2018;96:473 -83. doi:https://doi.org/10.1016/j.ijepes.2017.09.042.

[20] Srinivas MS. Distribution load flows: a brief review. In: Power Engineering Society Winter Meeting, 2000. IEEE; vol. 2. 2000, p. 942-945 vol.2. doi:10.1109/PESW.2000.850058.

[21] Balamurugan K, Srinivasan D. Review of power flow studies on distribution network with distributed generation. In: Power Electronics and Drive Systems (PEDS), 2011 IEEE Ninth International Conference on. 2011, p. 4117. doi:10.1109/PEDS.2011.6147281.

[22] Jenkins N, Allan R, Crossley P, Kirschen D, Strbac G. Embedded Generation (Power \& Energy Ser. 31). INSPEC, Inc.; 2000. ISBN 0852967748.

[23] Teng JH. A direct approach for distribution system load flow solutions. IEEE Transactions on Power Delivery 2003;18(3):882-7. doi:10.1109/TPWRD.2003.813818.

[24] Kersting W. Radial distribution test feeders. IEEE Trans Power Syst 1991;6(3):975-85. doi:10.1109/59.119237.

[25] AS4777.1: Grid connection of energy systems via inverters. Tech. Rep.; Standards Australia; 2016.

[26] AS60038: Standard voltages. Tech. Rep.; Standards Australia; 2000.

[27] Afandi I, Ciufo P, Agalgaonkar A, Perera S. A combined mv and lv network voltage regulation strategy for the reduction of voltage unbalance. In: Harmonics and Quality of Power (ICHQP), 2016 17th International Conference on. 2016, p. 318-23. doi:10.1109/ICHQP.2016.7783369. 